J. Indones. Math. Soc.

Vol. 27, No. 01 (2021), pp. 123-129.

\title{
ON SOFT b-w-OPEN SETS
}

\author{
Carlos Granados \\ Universidad del Atlántico, Barranquilla-Colombia, \\ carlosgranadosortiz@outlook.es
}

\begin{abstract}
In this paper, it used the notions of soft $\omega$-open sets to introduce and study the concepts of soft $b$ - $\omega$-open sets. Moreover, some of their properties are shown. Furthermore, the concepts of soft $b-T_{1}$ and soft $b-T_{2}$ spaces are defined. Also, it studies the relationships between the $b-\omega$-open sets of a given indexed family of topological spaces and the soft $b$ - $\omega$-open sets of their generated soft topological space. Besides, With these concepts it might study new notions related to this such as soft Lindelof or soft weakly Lindelof in soft topological spaces.
\end{abstract}

Key words and Phrases: Soft $b-\omega$-open sets, Soft $b-T_{1}$ spaces, Soft $b-T_{2}$ spaces.

\section{INTRODUCTION}

The notion of soft set was introduced by Molodtsov in 1999 [16], this concept is applied in several fields such that engineering, medical science, social science, etc. Besides, the concept of soft set has been grown by several researchers (see $[15,14]$ ), Chen is ones of those authors who had studied this concept, they introduced the notion of semi-open soft set [7]. For a soft topological space $(X, \tau, A)$ and $(F, A) \in$ $S S(X)_{A}$, then $(F, A)$ is called semi-open soft set if $(F, A) \tilde{\subseteq} C l(\operatorname{Int}(F, A))$. Besides, they proved some of their properties. On the other hand, Hdeib [13] introduced the notion of $\omega$-closed sets as a weaker notion of closed set by: Let $(X, \tau)$ be a topological space and $B \subset X$. A point $x \in X$ is called a condensation point of $B$ if for each open set $V$ with $x \in V$, the set $V \cap B$ is uncountable. $B$ is said to be $\omega$-closed if it contains all its condensation points. Many mathematicians have studied the concept of $\omega$-closed sets in different fields of the general topology (see $[12,6])$. Recently, Al Ghour and Hamed introduced and studied the notion of soft $\omega$-open sets and they proved some of their properties.

In this paper, motivated by the authors mentioned above, we define the concepts of soft $b$ - $\omega$-open sets by using the notion of soft $\omega$-open sets. Besides, we show

2020 Mathematics Subject Classification: 54A40, 54A10, 54A05.

Received: 13-06-2020, accepted: 01-12-2020. 
some of their properties. Furthermore, we define the concepts of soft $b-T_{1}$ and soft $b-T_{2}$ spaces.

\section{SOFT $b-\omega$-OPEN SETS}

In this section, we introduce and study the notions of soft $b$ - $\omega$-open sets by using the concept of $\omega$-set.

Definition 2.1. [8] Let $G \in S S(X, A)$. Then $G$ is said to be a countable soft set if for all $a \in A$, the set $G(a)$ is countable. The collection of all countable soft sets from $S S(X, A)$ will be denoted by $\operatorname{CSS}(X, A)$.

Definition 2.2. [8] Let $F \in S S(X, A) . F$ is called a soft point over $X$ relative to $A$ if there exit $e \in A$ and $x \in X$ such that

$$
F(a)= \begin{cases}\{x\} & \text { if } a=e \\ \emptyset & \text { if } a \neq e\end{cases}
$$

We denote $F$ by $e_{x}$. The family of all soft points over $X$ relative to $A$ is denoted by $\operatorname{SP}(X, A)$.

Definition 2.3. Let $(X, \tau, A)$ be a soft topological space and let $G \in S S(X \tau)$. Then, $G$ is said to be soft $b$ - $\omega$-open if for all $a_{x} \tilde{\in} G$, there exits a b-open $F \in \tau$ and $H \in C S S(X, A)$ such that $a_{x} \tilde{\in} F-H \subseteq \tilde{\subseteq} G$. The collection of all soft $b$ - $\omega$-open sets in $(X, \tau, A)$ will be denoted by $\tau_{b \omega}$.

Theorem 2.4. Let $(X, \tau, A)$ be a soft topological space and let $G \in S S(X, A)$. Then, $G$ is soft $b$ - $\omega$-open if and only if for every $a_{x} \tilde{\in} G$ there exits a b-open $F \in \tau$ such that $a_{x} \tilde{F}$ and $F-G \in C S S(X, A)$.

Proof. Necessary: Suppose that $G$ is soft $b$ - $\omega$-open and let $a_{x} \tilde{\in} G$, then there exits a $b$-open $F \in \tau$ and $H \in C S S(X, A)$ such that $a_{x} \tilde{\in} F-H \tilde{\subseteq} G$. Hence, $a_{x} \tilde{\in} F \in \tau$. Besides, since $F-H \subseteq \tilde{\simeq} G$, we have that $F-G \tilde{\simeq} H$ and so $F-G \in C S S(X, A)$.

Sufficiency: Suppose that for every $a_{x} \tilde{\in} G$, then there exits a $b$-open $F \in \tau$ such that $a_{x} \tilde{\in} F$ and $F-G \in C S S(X, A)$. Let $a_{x} \tilde{\in} G$. Then, there exits a $b$-open $F \in \tau$ such that $a_{x} \tilde{\in} F$ and $F-G \in C S S(X, A)$. Choose $H=F-\left(G \tilde{\cup} a_{x}\right)$. Therefore, $H \in C S S(X, A)$ and $a_{x} \tilde{\in} F-H \tilde{\subseteq} G$. Indeed, $G$ is soft $b$ - $\omega$-open.

Remark 2.5. For a soft topological space $(X, \tau, A)$, we will denote the collection of $\{F-H: F \in \tau$, where $F$ is a b-open and $H \in C S S(X, A)\}$ by $\tau_{b c}$.

Definition 2.6. [15] Let $F \in S S(X, A)$. Then, the following statements hold:

(1) $F$ is called null soft set over $X$ relative to $A$, denoted by $0_{A}$, if $F(a)=\emptyset$ for each $a \in A$.

(2) $F$ is called an absolute soft over $X$ relative to $A$, denoted by $1_{A}$, if $F(a)=X$ for each $a \in A$.

Theorem 2.7. Let $(X, \tau, A)$ be a soft topological space. Then, the following statements hold: 
(1) $\tau \subseteq \tau_{b c} \subseteq \tau_{b \omega}$.

(2) $\left(X, \tau_{b \omega}, A\right)$ is a soft topological space.

(3) $\tau_{b c}$ is a base for $\tau_{b \omega}$.

(4) Countable soft sets are soft semi-closed in $\left(X, \tau_{b c}, A\right)$

Proof. The following are details of the proof.

(1) Since $0_{A} \in C S S(X, A)$, then $\tau=\left\{F-0_{A}: F \in \tau\right.$ and $F$ is $b$-open $\} \subseteq \tau_{b c}$. Now, $\tau_{b c} \subseteq \tau_{b \omega}$ is clear.

(2) We will proof that $\left(X, \tau_{b \omega}, A\right)$ is a soft topological space, then:

(a) Since $0_{A}, 1_{A} \in \tau$ and by part (1) of this Theorem, $0_{A}, 1_{A} \in \tau_{b \omega}$.

(b) Let $F, G \in \tau_{b \omega}$ and let $a_{x} \tilde{\in} F \tilde{\cap} G$. Then, we have that $a_{x} \tilde{\in} F$ and $a_{x} \tilde{\in} G$. Then, by the Theorem 2.4, there exist $b$-open sets $H, W \in \tau$ such that $a_{x} \tilde{\in} H \tilde{\cap} W \in \tau$ and $H-F, W-G \in C S S(X, A)$. So, we can see that $(H \tilde{\cap} W)-(F \tilde{\cap} G)$. In consequence, by the Theorem 2.4, $F \tilde{\cap} G \in \tau_{b \omega}$.

(c) Let $\left\{G_{\delta}: \delta \in \Delta\right\} \subseteq \tau_{b \omega}$ and let $a_{x} \tilde{\in} \bigcup_{\delta \in \Delta} G_{\delta}$. Then, there is $\alpha \in \Delta$ such that $a_{x} \tilde{\in} G_{\alpha}$. Indeed, there exist $b$-open $F \in \tau$ and $H \in C S S(X, A)$ such that $a_{x} \tilde{\in} F-H \tilde{\subseteq} G_{\alpha} \tilde{\subseteq} \bigcup_{\delta \in \Delta} G_{\delta}$. Therefore, $\bigcup_{\delta \in \Delta}^{\tilde{G}} G_{\delta} \in \tau_{b \omega}$.

(3) This proof is clear.

(4) The proof is followed by part (1) of this theorem by the fact $\tau_{b c} \subseteq \tau_{b \omega}$.

Definition 2.8. [2] Let $(X, \tau, A)$ be a soft topological space. Then, $\left\{0_{A}\right\} \cup\left\{1_{A}-H\right.$ : $H \in C S S(X, A)\}$ is called the cocountable soft topology and is denoted by $\operatorname{coc}(X, A)$.

Proposition 2.9. For any soft topological space $(X, \tau, A), \operatorname{coc}(X, A) \subseteq \tau_{b c}$.

Proof. The proof is followed by the Remark 2.5 and Definition 2.8 .

Theorem 2.10. Let $(X, \tau, A)$ be a soft topological space. Then, the following statements are equivalent:

(1) $\operatorname{coc}(X, A) \subseteq \tau$.

(2) $\tau=\tau_{b c}$.

(3) $\tau=\tau_{b \omega}$.

Proof. (1) $\Rightarrow(2)$ : Suppose that $\operatorname{coc}(X, A) \subseteq \tilde{\tilde{\tau}} \tau$. We have to show that $\tau_{b c} \subseteq \tau$. Now, let $G \in \tau$ a $b$-open and $H \in C S S(X, A)$. Then, $G-H=G \tilde{\cap}\left(1_{A}-H\right)$. Since $\operatorname{coc}(X, A) \subseteq \tau$ this implies that $1_{A}-H \in \tau$, where $1_{A}$ is $b$-open and so $G-H \in \tau$. Hence, $\tau_{b c} \subseteq \tau$.

(2) $\Rightarrow(3)$ : Suppose that $\tau=\tau_{b c}$. Then, $\tau_{b c}$ is a soft topology. Now, by the Theorem 2.7 part (3), we have that $\tau_{b c}=\tau_{b \omega}$ and then $\tau=\tau_{b \omega}$.

(3) $\Rightarrow(1)$ : Suppose that $\tau=\tau_{b \omega}$. Then by the Proposition 2.9 and Theorem 2.7 part (1), we have that $\operatorname{coc}(X, A) \subseteq \tau_{b c} \subseteq \tau_{b \omega}=\tau$.

Proposition 2.11. Let $X$ be an initial universe and $A$ be a set of parameters. Then, $(\operatorname{coc}(X, A))_{b \omega}=\operatorname{coc}(X, A)$. 
Proof. The proof follows.

Theorem 2.12. For any soft topological space $(X, \tau, A), \tau_{b \omega}=\left(\tau_{b \omega}\right)_{b \omega}$.

Proof. By the Proposition 2.9 and Theorem 2.7 part (1), $\operatorname{coc}(X, A) \subseteq \tau_{b c} \subseteq \tau_{b \omega}$. Then, by the Theorem 2.10 , we have that $\tau_{b \omega}=\left(\tau_{b \omega}\right)_{b \omega}$.

Theorem 2.13. Let $(X, \tau, A)$ and $(X, \sigma, A)$ be two soft topological spaces. If $\tau \cup$ $\operatorname{coc}(X, A) \subseteq \sigma$, then $\tau_{b c} \subseteq \sigma$.

Proof. Let $F-H \in \tau_{b c}$, where $F \in \tau$ is a $b$-open set and $H$ is a countable soft set. Now, since $F \in \tau, 1_{A}-H \in \operatorname{coc}(X, A)$ and $\tau \cup \operatorname{coc}(X, A) \subseteq \sigma$. Indeed, $F, 1_{A}-H \in \sigma$ and then $F \tilde{\cap}\left(1_{A}-H\right)=F-H \in \sigma$

Lemma 2.14. Let $(X, \tau, A)$ and $(X, \sigma, A)$ be two soft topological spaces. If $\tau \cup$ $\operatorname{coc}(X, A) \subseteq \sigma$, then $\tau_{b \omega} \subseteq \sigma$.

Proof. The proof is followed by the Theorems 2.13 and 2.7 part (3).

Lemma 2.15. [17] Let $(X, \tau, A)$ be a soft topological space and let $\beta$ be a soft base for $\tau$. Then, for every $a \in A$, the family $\{F(a): F \in \beta\}$ forms a base for the topology $\tau_{a}$ on $X$.

Theorem 2.16. Let $(X, \tau, A)$ be a soft topological space. Then, for all $a \in A$, $\left(\tau_{a}\right)_{b \omega}=\left(\tau_{b \omega}\right)_{a}$.

Proof. Let $a \in A$. We have to show that $\left(\tau_{a}\right)_{b \omega} \subseteq\left(\tau_{b \omega}\right)_{a}$, it will be enough if we show that $U-C \in\left(\tau_{b \omega}\right)_{a}$ for all $U \in \tau_{a}$ and a countable subset $C \subseteq X$. Now, let $U \in \tau_{a}$ and let $C$ be a countable subset of $X$. Since $U \in \tau_{a}$, then there is a $b$-open $F \in \tau$ such that $F(a)=U$. Let $H=a_{C}$, then $H \in C S S(X, A)$. So, we have that $F-H \in \tau_{b \omega}$, and then $(F-H)(a)=F(a)-H(a)=U-C \in\left(\tau_{b \omega}\right)_{a}$.

Now, we have to show that $\left(\tau_{b \omega}\right)_{a} \subseteq\left(\tau_{a}\right)_{b \omega}$. By the Theorem 2.7 and Lemma 2.15 , we only have to prove that $\{(F-H)(a): F \in \tau$, where $F$ is $b$-open and $H \in C S S(X, A)\} \subseteq\left(\tau_{a}\right)_{b \omega}$. Let $F \in \tau$, where $F$ is $b$-open and $H \in C S S(X, A)$, then $(F-H)(a)=F(a)-H(a)$ with $F(a) \in \tau_{a}$ and $H(a)$ is a countable set of $X$ and this implies that $(F-H)(a) \in\left(\tau_{a}\right)_{b \omega}$.

Proposition 2.17. Let $(X, \tau, A)$ be a soft topological space. If $G \in \tau_{b \omega}$, then for all $a \in A, G(a) \in\left(\tau_{a}\right)_{b \omega}$.

Proof. Let $G \in \tau_{b \omega}$ and let $a \in A$. Then, $G(a) \in\left(\tau_{b \omega}\right)_{a}$ and by the Theorem 2.16, $G(a) \in\left(\tau_{a}\right)_{b \omega}$.

Definition 2.18. [1] Let $X$ be an initial universe and let $A$ be a set of parameters. Let $\left\{F_{a}: a \in A\right\}$ be an indexed family of topologies on $X$. Then the soft topology $\left\{F \in S S(X, A): F(a) \in F_{a}\right.$ for all $\left.a \in A\right\}$ will be denoted by $\bigoplus_{a \in A}\left(F_{a}\right)$. 
Lemma 2.19. [1] Let $X$ be an initial universe and let $A$ be a set of parameters. Let $\left\{F_{a}: a \in A\right\}$ be an indexed family of topologies on $X$. If $\beta_{a}$ is a base for $F_{a}$ for all $a \in A$. Then, $\left\{a_{Y}: a \in A\right.$ and $\left.Y \in \beta_{a}\right\}$ is a soft base of $\bigoplus_{a \in A} F_{a}$.

Theorem 2.20. Let $X$ be an initial universe and let $A$ be a set of parameters. Let $\left\{F_{a}: a \in A\right\}$ be an indexed family of topologies on $X$. Then, $\left(\bigoplus_{a \in A} F_{a}\right)_{b \omega}=$ $\bigoplus_{a \in A}\left(F_{a}\right)_{b \omega}$

Proof. We begin proving that $\left(\bigoplus_{a \in A} F_{a}\right)_{b \omega} \subseteq \bigoplus_{a \in A}\left(F_{a}\right)_{b \omega}$. By the Theorem 2.7 part (3), it will be enough showing $\left(\bigoplus_{a \in A} F_{a}\right)_{b c} \subseteq \bigoplus_{a \in A}\left(F_{a}\right)_{b \omega}$. Now, let $F \in \bigoplus_{a \in A} F_{a}$, where $f$ is $b$-open and $H$ is a countable soft set. Then, for every $a \in A, f(a) \in F_{a}$ and $H(a)$ is a countable subset of $X$ and then $(f-H)(a)=f(a)-H(a) \in\left(F_{a}\right)_{b \omega}$. Indeed, $F-H \in \bigoplus_{a \in A}\left(F_{a}\right)_{b \omega}$ for every $a \in A,\left\{U-C: U \in F_{a}\right.$ and $C$ is a countable subset of $X\}$ is a base for $\left(F_{a}\right)_{b \omega}$, by the Lemma 2.19, $\left\{a_{U-C}: a \in A, U \in F_{a}\right.$ and $C$ is countable subset of $X\}$ is a soft base for $\bigoplus_{a \in A}\left(F_{a}\right)_{b \omega}$. Hence, if we will prove that $\bigoplus_{a \in A}\left(F_{a}\right)_{b \omega} \subseteq\left(\bigoplus_{a \in A} F_{a}\right)_{b \omega}$, it is sufficient to show that $\left\{a_{U-C}: a \in A, U \in F_{a}\right.$ and $C$ is a countable subset of $X\} \subseteq\left(\bigoplus_{a \in A}\left(F_{a}\right)_{b \omega}\right.$. Now, we can see that $\left\{a_{U-C}\right.$ : $a \in A, U \in F_{a}$ and $C$ is a countable subset of $\left.X\right\}=\left\{a_{U-C}-a_{C}: a \in A, U \in F_{a}\right.$ and $C$ is a countable subset of $X\}$, and so, this ends the proof.

Lemma 2.21. [1] If $(X, F)$ is a topological space and $A$ is any set of parameters, then $(\tau(F))_{a}=F$ for all $a \in A$.

Proposition 2.22. If $(X, F)$ is a topological space and $A$ is any set of parameters, then $(\tau(F))_{b \omega}=\tau\left(F_{b \omega}\right)$ for all $a \in A$.

Proof. For each $a \in A$, the set $F_{a}=F$. Then, $\tau(F)=\bigoplus_{a \in A} F_{a}$ and by the Theorem 2.20, we have that:

$$
\begin{gathered}
(\tau(F))_{b \omega}=\left(\bigoplus_{a \in A} F_{a}\right)_{b \omega} \\
=\bigoplus_{a \in A}\left(F_{a}\right)_{b \omega} \\
=\tau\left(F_{b \omega}\right) .
\end{gathered}
$$


Definition 2.23. Let $(X, \tau, A)$ be a soft topological space. Then, $(X, \tau, A)$ is said to be soft b-p-space if the countable intersection of soft b-open sets is soft b-open.

Definition 2.24. Let $(X, \tau, A)$ be a soft topological space. Then, $(X, \tau, A)$ is said to be soft $b-T_{1}$ if for any two soft points $a_{x}, a_{y} \in S P(X, A)$ with $x \neq y$, there exist $b$-open sets $G, F \in \tau$ such that $a_{x} \tilde{\in} G-F$ and $a_{y} \tilde{\in} F-G$.

Lemma 2.25. A soft topological space $(X, \tau, A)$ is soft $b-T_{1}$ if for every soft point $a_{x} \in S P(X, A)$ is soft b-closed.

Proof. The proof is followed by the Definition 2.24

Theorem 2.26. If $(X, \tau, A)$ is soft $b-T_{1}$ and soft b-p-space, then $\tau=\tau_{b \omega}$.

Proof. By the Theorem 2.7 part (1), $\tau \subseteq \tau_{b \omega}$. Now, to show that $\operatorname{tau}_{b \omega} \subseteq \tau$, by the Theorem 2.7 part (3), it is enough to show that $\tau_{b c} \subseteq \tau$. Let $F \in \tau$, where $F$ is $b$-open and let $\operatorname{HCSS}(X, A)$. Since $(X, \tau, A)$ is soft $b-T_{1}$, then by the Lemma 2.25, $a_{x}$ soft closed for all $a_{x} \tilde{\in} H$, and so $F-a_{x} \in \tau$ for all $a_{x} \tilde{\in} H$, where $F-a_{x}$ is $b$-open. Since $(X, \tau, A)$ is soft $b-T_{1}$, then $\bigcap_{a_{x} \in H}\left(F-a_{x}\right) \in \tau$ is $b$-open. Therefore, $F-H=\bigcap_{a_{x} \in H}\left(F-a_{x}\right)$.

Lemma 2.27. For any soft topological space $(X, \tau, A) .\left(X, \tau_{b \omega}, A\right)$ is soft $b-T_{1}$.

Proof. The proof is followed by the Theorem 2.7 part (4) and Lemma 2.25.

Definition 2.28. Let $(X, \tau, A)$ be a soft topological space. Then, $(X, \tau, A)$ is said to be soft $b-T_{2}$ if for two soft points $a_{x}, a_{y} \in S P(X, A)$ with $x \neq y$, there exit b-open sets $G, F \in \tau$ such that $a_{x} \tilde{\in} G, a_{y} \tilde{\in} F$ and $G \tilde{\cap} F=0_{A}$.

Theorem 2.29. If $(X, \tau, A)$ is a soft topological space and $b-T_{2}$. Then, $\left(X, \tau_{b \omega}, A\right)$ is soft $b-T_{2}$.

Proof. Let $a_{x}, a_{y} \in S P(X, A)$ with $x \neq y$. Since $(X, \tau, A)$ is soft $b-T_{2}$, then there exist $b$-open sets $G, F \in \tau$ such that $a_{x} \tilde{\in} G, a_{y} \tilde{\in} F$ and $G \tilde{\cap} F=0_{A}$. Now, by the Theorem 2.7 part (1), $\tau \subseteq \tau_{b \omega}$ and hence $G, F \in \tau_{b \omega}$ an this ends the proof. Therefore, $\left(X, \tau_{b \omega}, A\right)$ is soft $b-T_{2}$.

\section{REFERENCES}

[1] Al Ghour, S., and Bin-Saadon, A., "On some generated soft topological spaces and soft homogeneity", Heliyon, 5(2019), e02061.

[2] Al Ghour, S., and Hamed, W., "On two classes of soft sets in soft topological spaces", Symmetry, 12(2020), 265-287.

[3] Al-shami, T.M., "Soft somewhere dense sets on soft topological spaces", Communications of the Korean Mathematical Society, 33:4 (2018), 1341-1356. 
[4] Al-shami, T.M., and Kocinac, L.D.R., "The equivalence between the enriched and extended soft topologies", Applied and Computational Mathematics, 18:2(2019), 149-162.

[5] Al-shami, T.M., and El-Shafei, M.E., "Partial belong relation on soft separation axioms and decision-making problem, two birds with one stone", Soft Computing, 24(2020), 5377-5387.

[6] Carpintero, C., Rosas, E., and Hussan, S., " $(i, j)-\omega$-semiopen sets and $(i, j)$ - $\omega$-semicontinuity in bitopological spaces", CUBO A mathematical Journal, 17:3(2015), 43-51.

[7] Chen, B., "Soft semi-open sets and related properties in soft topological spaces", Applied Mathematics and Information Sciences, 7(2013), 287-294.

[8] Das, S., and Samanta, S., "Soft metric", Ann. Fuzzy Math. Inform., 6(2013), 77-94.

[9] M. El-Shafei, E., Abo-Elhamayel, M., and Al-shami, T.M.," Partial soft separation axioms and soft compact spaces", Filomat, 32:13(2018), 4755-4771.

[10] El-Shafei, M.E., and Al-shami, T.M., "Applications of partial belong and total non-belong relations on soft separation axioms and decision-making problem", Computational and Applied Mathematics, 39:3(2020), https://doi.org/10.1007/s40314-020-01161-3.

[11] El-Sheikh, S., and A. El-Latiff, A., "Characterization of b-open soft sets in soft topological spaces", Journal of new Theory. 2(2015), 8-18.

[12] Granados, C., " $\omega-\mathcal{N}$ - $\alpha$-open sets and $\omega-\mathcal{N}-\alpha$ continuity in bitopological spaces", General Letters in Mathematics., 8:2(2020), 41-50.

[13] Hdeib, H., "w-closed mappings", Rev. Colombiana Mat., 16(1982), 65-78.

[14] Kharal, A., and Ahmad, B., "Mappings of soft classes", New Math. Nat. Comput., 7(2011), 471-481.

[15] Maji, P., Biswas, R., and Roy, A., "Soft set theory", Comput. Math. Appl., 45(2003), 555-562.

[16] Molodtsov, D., "Soft set theory-first results", Global optimization, control and games. III. Comput. Math. Appl. 37(1999), 19-31.

[17] Terepeta, M., "On separation axioms and similarity of soft topological spaces", Soft Comput., 23(2019), 1049-1057. 